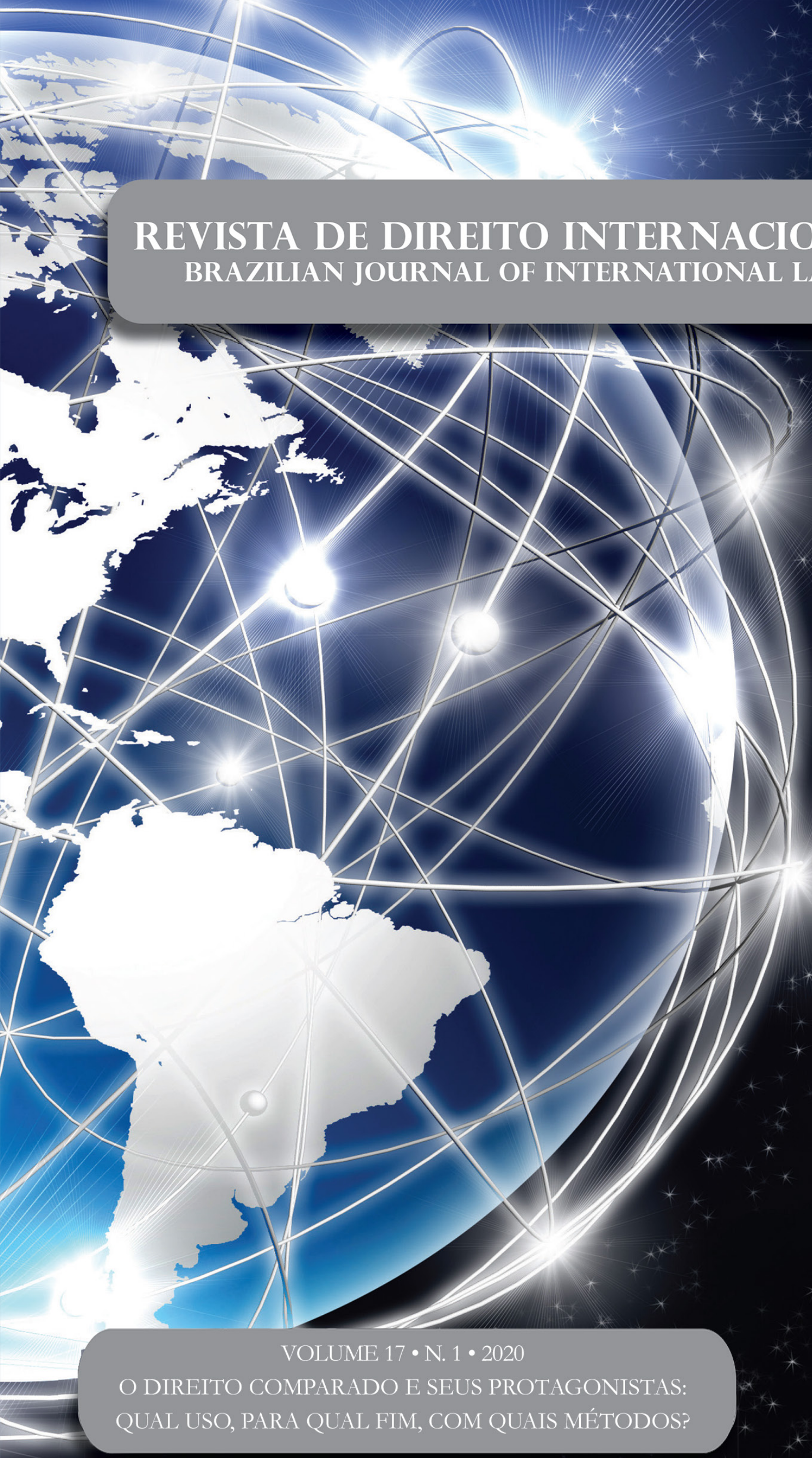

Tecnologias digitais e 0 comércio de bens e serviços na OMC/Digital

Technologies and the trade in goods and services in the WTO 
Parte I - O Direito comparado e seus Protagonistas: QuAL uso, PARA QUAL FIM, COM QUAIS MÉTODOS? .....................................................................1

EDITORIAL ............................................................................................................... 3

"Tudo o que precisamos fazer é ter certeza de que continuaremos conversando".............................. 3

Gustavo Cerqueira e Patrícia Perrone Campos Mello

Direito comparado E METOdologia $\quad$................................................... 6

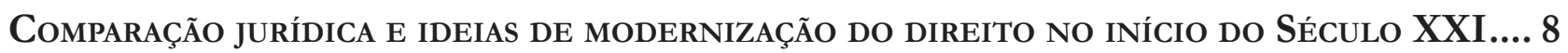
Gustavo Cerqueira

O DIREITO COMPARADO: ESFORÇO DE RESGATE HISTORIOGRÁFICO E DE PROBLEMAS METODOLÓGI$\cos$

Arnaldo Sampaio de Moraes Godoy e Gustavo Fereira Ribeiro

Direito Comparado e Política: Reflexões Necessárias .42

Raphael Carvalho de Vasconcelos e Deo Campos Dutra

Direito comparado no Brasil

L'originalité du Droit Brésilien et le Droit Comparé .57

Arnoldo Wald

LEI DA BOA RAZÃo E COMPARATISMO JURÍDICO NA DOUTRINA CIVILISTA BRASILEIRA DE 1850 A 1880

Alan Wruck Garcia Rangel

O STF EM REDE? QUANTO, COMO, COM QUE ENGAJAMENTO ARGUMENTATIVO O STF USA PRECEDENTES ESTRANGEIROS EM SUAS DECISÕES?

Patrícia Perrone Campos Mello e Felipe Meneses Graça 
Suprema Imprecisão: a metodologia em Direito Constitucional Comparado E as deficiênCias Em Seu uso pelo Supremo Tribunal Federal

Alonso Freire e Hugo Sauaia

Direitos comparados

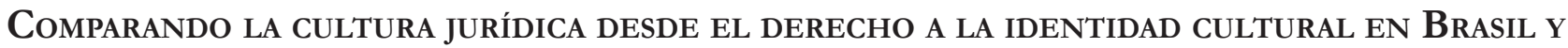

CHILE 145 Juan Jorge Faundes e Fabian Le Bonniec

O Divino e o Racional no Direito: notas para um diálogo entre sistemas jurídicos 181 Salem Hikmat Nasser e José Garcez Ghirardi

Regulação do discurso de Ódio: análise comparada em países do Sul Global 196 Jane Reis Gonçalves Pereira, Renan Medeiros de Oliveira e Carolina Saud Coutinho

Parte II - Outros temas

LA OTRA CARA DE LA MONEDA: PROTECCIÓN CONSTITUCIONAL DE LA EMPRESA, EL EMPRENDIMIENTO Y LA LIBRE COMPETENCIA EN CHILE y COLOMBiA

Juan Pablo Díaz Fuenzalida e Juan Sebastián Villamil Rodríguez

The European Court of Human Rights Decision on there 'Burqa Ban'and the CritiCal analysis of the Pragmatic experimental logic 258 Flavianne Fernanda Bitencourt Nóbrega e George Browne Rego

Direitos humanos das deslocadas ambientais e os impactos da Usina de Belo Monte: da EXPLORAÇÃO AMAZÔNICA À SUBJUGAÇÃO FEMININA

Thais Silveira Pertille e Letícia Albuquerque

Solução de Controvérsias em Acordos de Investimento: as experiênCias do CPTPP, CETA E DOS ACFIs

Fábio Morosini, Vivian Daniele Rocha Gabriel e Anastacia Costa

50 anos dos “direitos da Criança” na Convenção Americana de Direitos Humanos: a HISTÓRIA DO ARTIGO 19 311

Sven Peterke e Paloma Leite Diniz Farias 
EMPRESAS E DIREITOS HUMANOS: COMPARTILHANDO VALOR E RESPONSABILIDADES .325 Melina Girardi Fachin

CONTEMPORARY RESPONSES TO BUSINESSES' NEGATIVE HUMAN RIGHTS IMPACT 341 Andres Felipe Lopez

Human Right to LABOR PROTECTION IN UKRAINE: CURRENT SITUATION AND THE PROSPECTS OF IMPLEMENTATION OF INTERNATIONAL RULES 363 Nina Daraganova

International Regulation and Global Governance: The EU influential Method IN TIMES OF NORMATIVITY CHANGE 373 Gabriela Hühne Porto, Paula Wojcikiewicz Almeida e Juliana Maia F. A. Netto

Tecnologias digitais e o comércio de bens E SERviços na OMC/Digital. 391 Umberto Celli Junior

Los fuertes hacen lo QUe PUeden: exponiendo los límites de la Corte Penal InternaCIONAL .406

Cristián D. González-Ruiz e Víctor M. Mijares

Human Rights, Humanitarian Law and State Power 418 Renata Nagamine e João Roriz

Regional integration in the South Pacific: Challenges for Public governance .433 Joanna Siekiera

Parte III - Resenhas 443

RESENHA DA OBRA

“Democracia e policentrismo do poder", de Murilo Gaspardo 445 Angela Limongi Alvarenga Alves 


\section{Tecnologias digitais e o comércio de bens e serviços na OMC/Digital*}

\section{Technologies and the trade in goods and services in the WTO}

Umberto Celli Junior**

\section{Resumo}

O comércio global será cada vez mais realizado por meio de plataformas digitais, o que resultará no surgimento de novos meios de entrega de produtos/bens e modos de serviços. Isso impõe enormes desafios à sua regulação no âmbito da Organização Mundial do Comércio (“OMC”), especialmente em razão da natureza ainda difusa e não totalmente compreendida do ambiente digital e do constante processo de evolução das tecnologias digitais. Ainda que se leve em conta uma definição mais ampla que permite classificar as transações do comércio digital ou eletrônico pelos meios e pelas atividades que resultam na entrega de bens e serviços, é complicado vislumbrar, em toda sua dimensão, os impactos que tais transações comportam para o conjunto normativo da OMC, especialmente para dois de seus principais acordos, o Acordo Geral sobre Tarifas e Comércio ("GATT"), e o Acordo Geral sobre o Comércio de Serviços ("GATS”). Por essa razão, em vez de seguir esse tipo de classificação mais abrangente de comércio digital ou eletrônico, opta-se por uma análise que utiliza como fio condutor duas tecnologias digitais: a impressão em 3D e o blockchain. Com base nessa delimitação, este artigo apresenta as possibilidades e as dificuldades de enquadramento dessas duas tecnologias digitais no GATT e no GATS, sustentando que, além de sua necessária revisão e adaptação, eles deveriam ser complementados por outros acordos plurilaterais, também sujeitos à revisão e ampliação do número de participantes, como o Acordo sobre Tecnologia da Informação ("ITA"), no caso do GATT, e o Acordo sobre o Comércio de Serviços ("TISA"), no caso do GATS. Embora não exaustivas, as reflexões contidas neste artigo podem contribuir para o debate da imperiosa reforma da OMC em face da economia digital.

Palavras-chave: Comércio global. Tecnologias digitais. GATTT. GATS. Impressão em 3D. Blockchain.

* Recebido em 27/09/2019 Aprovado em 16/05/2020

** Professor Titular de Direito Internacional do Departamento de Direito Público da Faculdade de Direito de Ribeirão Preto da USP. Email: umbcelli@usp.br

\section{Abstract}

Global trade shall increasingly be carried out through digital platforms, which shall result in the emergence of new means of delivering products/ goods and modes of services. This poses huge challenges to its regulation within the World Trade Organization ("WTO”), especially given the still diffuse and not fully understood nature of the digital environment and the 
ever-evolving process of digital technologies. Although, based on a broader definition, it is possible to classify digital or electronic trade transactions through the means and activities that result in the delivery of goods and services, it seems to be still complicated to envisage, in all their dimension, the impacts such transactions have on the WTO's regulatory framework, especially on two of its main agreements, the General Agreement on Tariffs and Trade ("GATT") and the General Agreement on Trade in Services ("GATS"). Accordingly, rather than following this type of broader electronic or digital trade classification, the analysis made in this article focus on two digital technologies: the 3D printing and blockchain. Based on this delimitation, this article seeks to show the possibilities and difficulties of framing these two technologies in the GAT'T and the GATS, arguing that, in addition to necessarily revising and adapting them, they should be complemented by other plurilateral agreements, also subject to review and increase in number of participants, such as the Information Technology Agreement ("ITA"), in the case of the GATT, and the Trade in Services Agreement ("TISA"), in the case of the GATS. Albeit not exhaustive, the reflections contained in this article might contribute to the discussions on the imperative reform of the WTO in view of the digital economy.

Keywords - Global trade. Digital technologies. GATT' GATS. 3D printing. Blockchain.

\section{Introdução}

O rápido crescimento da economia digital, isto é, da aplicação de tecnologias baseadas na Internet à produção de bens e serviços, reformulou o modo de consumo, produção e comércio, transformou investimentos globais e criou mercados. Alguns denominam esse fenômeno de $4^{a}$ Revolução Industrial, Nova Revolução Industrial ou Nova Revolução Digital. Independentemente da definição mais adequada, o comércio será, cada vez mais, realizado por meio de plataformas digitais, novos meios de entrega de bens e produtos surgirão, assim como novos modos de serviços.

Trata-se de enormes desafios colocados pelas tecnologias digitais à regulação do comércio global e aos Estados. O sistema multilateral de comércio, cujo centro de gravidade, apesar de sua atual paralisia, ainda é a
OMC, estaria preparado para esses desafios? Se, de um lado, pode-se especular que princípios básicos desse sistema, tais como transparência, liberalização, cooperação e não discriminação, tendem a permanecer, de outro, sua adequação às novas tecnologias digitais parece ser uma questão em aberto, especialmente em face da natureza, ainda difusa e não totalmente compreendida, do ambiente digital e do constante processo de evolução dessas tecnologias.

O exame dessa questão impõe, em primeiro lugar, a necessidade de tentar-se delinear e sistematizar, com algum grau de precisão, algumas das principais características do que se entende por produto ou comércio digital.Ainda que se leve em conta uma definição mais ampla que permite classificar as transações do comércio digital ou eletrônico pelos meios e pelas atividades que resultam na entrega de bens e serviços, é complicado vislumbrar, em toda sua dimensão, os impactos que tais transações comportam para o conjunto normativo da OMC, especialmente para dois de seus principais acordos, o GATT e o GATS.

Por essa razão, em vez de seguir esse tipo de classificação mais abrangente de comércio digital ou eletrônico, opta-se com inspiração no excelente trabalho contido no World Trade Report 2018, da OMC ${ }^{1}$, por uma análise que utiliza como fio condutor duas tecnologias digitais: a impressão em 3D e o blockchain.

Com base nessa delimitação, este artigo apresenta as possibilidades e as dificuldades de enquadramento dessas duas tecnologias digitais no GATT e no GATS, sustentando que, além de sua necessária revisão e adaptação, eles deveriam ser complementados por outros acordos plurilaterais, também sujeitos à revisão e ampliação de seu número de participantes, tais como o ITA, no caso do GATT, e o TISA, no caso do GATS.

Para tanto, de início, são apresentados uma definição de produto ou comércio digital e alguns dos modos de transações que o caracterizam, passando-se em seguida ao exame da relação da impressão em 3D com o GATTT e o GATS e a erosão das fronteiras entre esses acordos. Na sequência, após o delineamento das principais características do blockchain, especula-se sobre como seu uso, associado ao comércio internacional, pode

WTO. WTO World Trade Report (2018) - The future of World Trade: how digital technologies are transforming global commerce. Disponível em: https://www.wto.org/english/res_e/publications_e/world_trade_ report18_e.pdf. Acesso em: 20 de out. 2018. 
estar relacionado às cadeias globais de suprimento, as quais envolvem, dentre outros aspectos, o fluxo de dados. Argumenta-se que, de alguma forma, as normas do GATS abrangeriam os fluxos de dados transfronteiriços e o comércio de serviços digitais. Daí ser o GATS de fundamental importância para o blockchain.

Nas considerações finais, destaca-se, como mencionado, a importância do ITA como acordo complementar ao GATT, desde que tenha seu escopo atualizado no sentido de incluir produtos digitais em sua lista de classificação. No tocante ao GATS, além de o TISA ser um interessante caminho paralelo, enfatiza-se que, em ambos os acordos ou mesmo em um eventual acordo plurilateral sobre comércio eletrônico, será importante deixar claro que não poderá haver restrições ao fluxo ou transferência internacional de dados, salvo em alguns casos excepcionais. Embora não exaustivas, as reflexões contidas neste artigo podem contribuir para o debate da imperiosa reforma da OMC em face da economia digital.

\section{Produto/Comércio digital}

Produto digital refere-se a qualquer bem ou serviço que, de alguma forma, torna-se disponível por meio do uso de tecnologia digital, a Internet. ${ }^{2}$ Comércio digital constitui a transmissão (transfronteiriça ou não) de bens e serviços por meios eletrônicos. Não há, ainda, uma classificação amplamente aceita dos modos de comércio que dependem dos meios digitais. Definições mais restritivas considerariam o comércio digital ou comércio eletrônico como aquelas transações que envolvem bens e serviços digitais. Definições mais amplas envolveriam transações comerciais de produtos físicos ou serviços em que as partes assinam um contrato usando plataformas digitais.

Com base em uma definição mais ampla, as transações de comércio digital ou eletrônico, segundo propõem Ciuriak e Ptashkina, poderiam ser classificadas em cinco modos dependendo dos meios de entrega e

\footnotetext{
2 Nos termos do art. 14.1 do Acordo de Livre Comércio entre a Coreia do Sul e Singapura de 2006, “““digital products” means computer programmes, text, video, images, sound recordings and other products that are digitally encoded, regardless of whether they are fixed on a carrier medium or transmitted electronically;". Disponível em: https://investmentpolicyhub.unctad.org/Download/TreatyFile/2709. Acesso em: 20 de out. 2018.
}

das atividades que se enquadram em cada um deles, a saber: (i) Modo 1 - do "digital para o real", incluindo provimento de acesso à Internet. Exemplos e modelos de negócio: mecanismos de busca na web, " $e$ -learning", aplicativos móveis, serviços de comunicação (WhatsApp ou Skype), serviços de informação (mapas e enciclopédias "on line"), propaganda "on line" e Netflix; (ii) Modo 2 - do "real para o real", como operações do tipo negócio-domicílio ("B2H”) e negócio-negócio ("B2B") com intermediação digital. Exemplos e modelos de negócio: Amazon e outros serviços de distribuição (B2B); serviços de viagem (reservas de hotel e de voos) e compra de software; (iii) Modo 3 - transações domicílio-domicílio ("H2H") do "real para o real", com intermediação digital. Exemplos e modelos de negócio: transações "peer-to-peer" (eBay,Uber, AirBnB); (iv) Modo 4 - transações H2H do "real para o real", com intermediação digital. Exemplos e modelos de negócio: plataformas de serviço como Fiverr e Upwork, que correspondem ao modo 4 do GATS - movimento de pessoas); e (v) Modo 5 - capitalização de fluxo de dados. Exemplos e modelos de negócio: comercialização de dados pessoais realizados pelo Facebook e pelo Google). ${ }^{3}$

Como se pode perceber, o Modo 1 refere-se a atividades nas quais os bens e os serviços são entregues do digital para o real (em linha com a definição mais estrita mencionada acima), enquanto os Modos 2, 3 e 4 descrevem aquelas atividades em que bens e serviços são entregues do real para o real, mas parte da transação ocorre por meio de plataformas digitais, inclusive a assinatura de contrato (definição mais abrangente). O Modo 5 envolve a capitalização de fluxo de dados em que as empresas comercializam dados pessoais gerados por seus usuários.

Apesar de essa taxonomia proporcionar uma compreensão da complexidade do comércio digital e mesmo de barreiras comerciais que poderão surgir, os próprios autores reconhecem algumas de suas limitações. Apenas como exemplo, o escopo do Modo 1, que se restringe a produtos digitais baixados/acessados via "streaming", contempla, unicamente, o comércio baseado em empre-

CIURIAK, Dan; PTASHKINA, Maria. Começaram as guerras no comércio digital: delineando os campos da batalha regulatória. Revista Pontes, vol. 14, n. ${ }^{\circ}$ 1, 2018. Disponível em: http://www.ictsd. org/bridges-news/pontes/news/come $\% \mathrm{C} 3 \% \mathrm{~A} 7$ aram-as-guerrasno-com $\%$ C3\%A9rcio-digital-delineando-os-campos-de-batalha. Acesso em: 29 de mar. 2019. 
sas, não em países. O Modo 2, que abrange o comércio de bens e serviços, viabilizado por meios digitais, é conduzido por meio do modelo de negócios adotado por empresas como a Amazon que concorre com outros modelos de distribuição estabelecidos. Em princípio, segundo eles, "esses fluxos podem ser mensurados com estatísticas tradicionais de comércio internacional, ainda que o uso desse modelo possa comprometer a qualidade da classificação do produto e levantar questões sobre a relevância dos acordos de livre comércio, já que estes não são desenhados com o fim de facilitar transações transfronteiriças de varejo". O Modo 3 elimina negócios tradicionais e desafia regimes regulatórios e tarifários estabelecidos, gerando questões ainda sem solução. Por sua vez, o Modo 5 é mais complicado ainda, uma vez que grande parte dos fluxos de dados não configura transações digitais (não há pagamento e nenhum registro em papel), mas é parte constitutiva e facilitadora do comércio digital e do comércio que depende de meios digitais em todos os quatro modos descritos acima. ${ }^{4}$

A partir dessa sistematização por modos, não parece ser tarefa fácil, portanto, determinar, na dimensão requerida, os impactos que as transações envolvidas comportam para o conjunto normativo da OMC, em especial para o GATT e o GATS.

Como destaca Nick Ashton-Hart, "[to] understand the Internet in a trade context is a challenge. This is partly because trade specialists are confronting a subject that is new to them, but also because those who advocate for networked economy provisions in trade often don't explain the fundamentals so that policymakers can relate the unfamiliar to the existing trading system they know". ${ }^{5}$

4 CIURIAK, Dan; PTASHKINA, Maria. Começaram as guerras no comércio digital: delineando os campos da batalha regulatória. Revista Pontes, vol. 14, n. ${ }^{\circ}$ 1, 2018. Disponível em: http://www.ictsd. org/bridges-news/pontes/news/come $\% \mathrm{C} 3 \% \mathrm{~A} 7$ aram-as-guerrasno-com $\%$ C3\%A9rcio-digital-delineando-os-campos-de-batalha. Acesso em: 29 de mar. 2019.

5 ASHTON-HART, Nick. Addressing the Networked Economy in Trade Policy. In: BRAGA, Carlos A.; HOEKMAN, Bernard (ed.). Future of the Global Trade Order. Florença, Lausanne, São Paulo: European University Institute, IMD, Fundação Dom Cabral, $2^{a}$ ed., 2017, p. 218. Nesse diapasão, em alentado estudo sobre contratos inteligentes ("smart contracts"), que utilizam a tecnologia blockchain, PARDOLESI, Roberto e DAVOLA, Antonio também ressaltam a dificuldade de sistematização e intepretação de conceitos que envolvem tecnologia, direito e regulação. Mesmo após terem feito a opção metodológica de restringir sua análise sob a perspectiva do direito e da regulação "excluding (on one hand) works by technicians generically dealing with regulatory topics and (on the other
O exame das tecnologias impressão em 3D e blockchain, feito nos próximos parágrafos, embora não exaustivo, permite relacionar, de alguma maneira, o que ainda não é totalmente familiar e de natureza difusa às regras do sistema de comércio existente e conhecido.

\section{Impressão em 3D: GATT ou GATS?}

Pode-se afirmar que, desde sua criação em 1995, a $\mathrm{OMC}$ vem tentando dar algum tipo de resposta às rápidas mudanças tecnológicas. Em 1996, durante a Conferência Ministerial de Singapura, 29 Membros firmaram o ITA (acordo plurilateral). Desde então, o número de participantes cresceu para 82, o que representa cerca de $97 \%$ do comércio mundial de produtos de tecnologia da informação. Seus participantes estão comprometidos com a eliminação completa de impostos de importação incidentes sobre esses produtos. $\mathrm{Na}$ Conferência Ministerial de Nairóbi, em 2015, mais 201 produtos foram adicionados à lista de liberalização, ou seja, de tarifa zero para importação ${ }^{6}$. Os produtos que compõem a lista são físicos, como semicondutores utilizados na indústria de tecnologia da informação, equipamentos de telecomunicações, computadores e softwares. O ITA não trata especificamente de produtos digitais.

O 'Work Programme sobre Comércio Eletrônico' também é um exemplo importante dos esforços da OMC. Adotado na Conferência Ministerial de Genebra, em 1998, visa estabelecer um abrangente programa de trabalho para examinar "all trade-related issues arising from global e-commerce" ${ }^{\text {"7 }}$, incluindo a proteção da priva-

one) contributions by legal scholars misinterpreting the inner characteristics of the technology, the task proved itself arduous. Note that this is another significant proof of how difficult it is to develop a true interdisciplinary approach to the topic, suggesting - once again - caution for those who want to engage in the area of Law \&Technology." PARDOLESI, Roberto; DAVOLA, Antonio. What is wrong in the debate about Smart Contracts, p. 3. Disponível em: https://papers.ssrn.com/sol3/papers.cfm?abstract_id=3339421. Acesso em: 5 de mai. 2020.

6 Dados da OMC. Disponíveis em: https://www.wto.org/English/tratop_e/inftec_e/inftec_e.htm. Acesso em: 26 mar.2019.

7 Disponível em : https://www.wto.org/english/thewto_e/ minist_e/mc11_e/briefing_notes_e/bfecom_e.htm. Acesso em: 26 de mar.2019. Nesse sentido, como também observado por RIBEIRO ALVES, Gleisse, de "acordo com a OMC, o termo "comércio eletrônico" envolveu uma ampla gama de atividades diferentes: como "meios de produção, distribuição, comercialização, venda ou entrega de bens ou serviços por meios eletrônicos". RIBEIRO ALVES, Gleisse. O Acordo GATS e sua Aplicação aos Serviços do 
cidade e o acesso e o uso das redes e serviços públicos de transporte de telecomunicações, dentre outros. É verdade que

[p]ractically speaking, the dedicated discussions on e-commerce made no headway on any of the selected (admittedly complex) focus areas, notably the classification of the content of certain electronic transmissions; development-related issues; fiscal implications of e-commerce; relationship (and possible substitution effects) between e-commerce and traditional forms of commerce; imposition of customs duties on electronic transmissions; competition; jurisdiction and applicable law and other legal issues. ${ }^{8}$

Posições do Órgão de Apelação manifestadas nos casos United States-Measures Affecting the Cross-Border Supply of Gambling and Betting Services, WT/DS285/AB/R (adotado em 7 abr. 2005) e China-Measures Affecting Trading Rights and Distribution Services for Certain Publications and Audiovisual Entertainment Products, WT/DS363/ $A B / \mathrm{R}$ (adotado em $21 \mathrm{dez}$. 2009) trazem alguma sinalização sobre o alcance dos produtos digitais.

Contudo, a fundamental questão envolvendo a distinção entre bens e serviços ainda não foi esclarecida, ou seja, os "Membros da OMC não chegaram a um acordo sobre a classificação do tipo de comércio realizado pela Internet: se ele é classificado como bem ou serviço." ${ }^{10}$

Comércio Eletrônico. Revista de Direito Internacional (Brazilian Journal of International Law), Vol. 12, n. ${ }^{\circ}$ 2, 2014, p. 323. A Organização para a Cooperação e Desenvolvimento Econômico (OCDE), por sua vez, reconhece que existe crescente consenso de que o comércio eletrônico ou digital "encompasses digitally-enabled transactions of trade in goods and services that can either be digitally or physically delivered [...]".OECD. The impact of digitalization on trade. Disponível em: https://www.oecd.org/trade/topics/digital-trade/. Acesso em: 8 de mai. 2020.

8 WUNSCH-VINCENT, Sacha; HOLD, Arno. Towards coherent rules for digital trade: Building on efforts in multilateral versus preferential trade negotiations. In: BURRI, Mira; COTTIER, Thomas (Orgs.). Trade Governance in the Digital Age. Cambridge: Cambridge University Press, 2012, p. 180. Mais informações sobre a evolução das negociações do 'Work Programme sobre o Comércio Eletrônico’ no parágrafo 4.1, abaixo.

9 Em ambos os casos, o Órgão de Apelação da OMC entendeu que as regras do GATS se aplicam, integralmente, a transações transfronteiriças baseadas na Internet (e-commerce) e serviços fornecidos eletronicamente. Também foi reconhecido o Princípio da Neutralidade da Rede, ou seja, de que o GATS é neutro tecnologicamente no sentido de que não contém qualquer disposição que faça distinção entre os variados meios tecnológicos de fornecimento de um serviço.

10 RIBEIRO ALVES, Gleisse. O Acordo GATS e sua Aplicação aos Serviços do Comércio Eletrônico. Revista de Direito Internacional (Brazilian Journal of International Law), Vol. 12, n. ${ }^{\circ}$ 2, 2014, p. 323-324. Ainda segundo a autora, além "da aplicação dos Acordos GATT e
Essa indefinição é percebida com clareza especialmente com relação a uma das categorias de produtos digitais, a impressão em 3D ou manufatura aditiva remota.

Dois tipos de produtos digitais, a saber "bens tangíveis encomendados pela Internet" e "serviço entregue eletronicamente", não oferecem dificuldades quanto a seu enquadramento. No primeiro, o exemplo clássico é a aquisição de um livro feito pela Internet no site da Amazon. Não há diferença de uma compra efetuada diretamente em uma livraria. Existe consenso de que esse livro ou qualquer outro produto tangível encomendado pela Internet é um bem para os propósitos da OMC. Nesse caso, se o vendedor e o comprador do livro estivessem localizados em países diferentes, a importação do livro seria abrangida pelo GATT.

Raciocínio similar seria aplicável aos serviços entregues eletronicamente. Não há dúvida de que um parecer jurídico enviado de um escritório de advocacia para uma empresa localizada em outro país seja um serviço para os fins do GATS.

Um pouco mais complicado é o caso de um terceiro tipo, a dos denominados "e-products", que são produtos baseados em conteúdo que antes eram entregues em forma tangível e que agora podem ser baixados pela Internet em formato eletrônico. Os "e-products" incluem, por exemplo, arquivos de música mp3, "e-books" ou filmes baixados digitalmente. A literatura especializada tem analisado a natureza do "e-product" no âmbito do GATT/GATS, mas não determinou, de modo mais objetivo, qual desses acordos deveria prevalecer. ${ }^{11}$

GATS, alguns Membros da OMC defendem que o comércio da Internet deve ser regulamentado também pelo Acordo TRIPS porque deve-se regulamentar a propriedade do bem e não a forma como o produto chegará ao consumidor." A autora observa também que as pesquisas desenvolvidas por grupos de trabalho da OMC sustentam que o GATS é o mais aplicável à regulamentação do comércio eletrônico, "uma vez que as transações realizadas na Internet são, na maioria dos casos, caracterizadas por serviços e que, entre os Acordos Constitutivos da OMC, o Acordo de Serviços - GATS é o mais adaptável." Seja como for, “[p]ending consensus on whether digital products are goods or services, WTO Members have reached an informal agreement not to impose customs duties on electronic transmissions (including transmissions of audiovisual products)". VOON, Tania. A New Approach to Audiovisual Products in the WTO: Rebalancing GATT and GATS. UCLA Entertainment Review, 14 (1), 2007, p. 9.

11 FLEUTER, Sam. The Role of Digital Products Under the WTO: A New Framework for GATT and GATS Classification. Chicago Journal of International Law, Vol. 17: No. 1, Article 5, 2016, p. 159. Disponível em: http://chicagounbound.uchicago.edu/cjil/vol17/ iss1/5. Acesso em: 5 de nov. 2018. 
Já a impressão em 3D é um produto digital mais complexo que requer um maior detalhamento técnico. Trata-se de "um processo de fabricação de um objeto sólido tridimensional de praticamente qualquer forma a partir de um modelo digital [...] obtido por meio de um processo aditivo, onde camadas sucessivas de material são colocadas em diferentes [...] formatos distintos das tradicionais técnicas de usinagem, que dependem principalmente da remoção de material por métodos como corte ou perfuração (processos subtrativos)". ${ }^{12}$ Tem, atualmente, ampla aplicação industrial como peças para aviões, trens e automóveis, dentre outras.

A General Electric, por exemplo, utiliza a impressão em 3D para turbinas de avião e equipamentos médicos. A Lockheed Martin e a Boeing 3D imprimem equipamento aeroespacial. Uma empresa chinesa desenvolveu um sistema para imprimir casas inteiras. ${ }^{13}$ Uma plataforma de comércio eletrônico, a Shapeways, permite que "designers" façam o carregamento ("upload") de projetos de produtos, usem a impressão em 3D para criar os itens físicos e gerenciem a logística para que esses itens cheguem aos consumidores finais. ${ }^{14}$ Estima-se que, em longo prazo, a impressão em 3D possa substituir os métodos tradicionais de fabricação, reduzindo a necessidade de produção e montagem terceirizada, o número de etapas de produção e a necessidade de estoque, armazenamento, distribuição, centros de varejo e embalagem.

A diferença com relação à impressão em cores $2 \mathrm{D}$ está no uso pela tecnologia 3D de um tipo especial de arquivo denominado "Computer-Aided Design" (“CAD”). Como qualquer outro arquivo digital, o CAD

\footnotetext{
12 WTO. WTO World Trade Report (2018) - The future of World Trade: how digital technologies are transforming global commerce, p. 10. Disponível em: https://www.wto.org/english/res_e/publications_e/world_ trade_report_e.pdf. Acesso em 20 de out. 2018.

13 FLEUTER, Sam. The Role of Digital Products Under the WTO: A New Framework for GATT and GATS Classification. Chicago Journal of International Law, Vol. 17: n. 1, Article 5, 2016, p. 158-159. Disponível em http://chicagounbound.uchicago.edu/cjil/ vol17/iss1/5. Acesso em 5 de nov. 2018.

14 WTO. WTO World Trade Report (2018) - The future of World Trade: how digital technologies are transforming global commerce, p. 32. Disponível em: https://www.wto.org/english/res_e/publications_e/world_ trade_report_e.pdf. Acesso em 20 de out. 2018. Observe-se também que cadeias de valor "em um mundo de impressão 3D difundida podem não apenas tornar-se mais curtas — com o surgimento de centros de produção próximos a grandes bases de clientes ou de centros de inovação - mas também podem ser muito diferentes, baseadas principalmente na troca de dados transfronteiriços na forma de projetos, projetos e software, e não na troca transfronteiriça de bens e serviços materiais", idem, p. 10.
}

pode ser transmitido além-fronteiras pela Internet.

Nesse caso, a questão que se coloca é se esse arquivo CAD deveria ser tratado como um bem ou serviço. Como determinar se o produto digital é bem ou serviço?

Com base em perspectiva formalista, a maneira menos complicada de determinar se um produto digital é um bem ou serviço seria por meio da verificação da definição desses termos no GATT e no GATS. Nenhum dos dois tem uma definição significativa. Enquanto o GATT não prevê qualquer definição de "bens", no GATS, "serviços" são definidos como qualquer serviço em qualquer setor exceto serviços prestados no exercício da autoridade governamental (art. I, 3, b) ${ }^{15}$ Como mencionado anteriormente, para produtos digitais em particular, nem os acordos da OMC nem o "Work Programme sobre Comércio Eletrônico" contêm definição para "comércio digital", "bens digitais" ou "serviços digitais".

Frequentemente, bens são considerados tangíveis e serviços intangíveis. No caso

Canada - Certain Measures Concerning Periodicals, o Órgão de Apelação entendeu que periódicos são um produto tangível de tinta e papel, o que os impediria de ser classificados como serviços. ${ }^{16}$ Essa distinção parece fornecer uma solução simples para produtos digitais. Um produto digital não é tangível, portanto, é um serviço. Porém, quando são considerados os "e-products", essa conclusão conduz ao estranho entendimento de que um $\mathrm{CD}$ seria classificado como um bem, enquanto a versão digital desse álbum um serviço. Com a impressão em 3D, essa distinção também produz resultados pouco satisfatórios. ${ }^{17}$

No âmbito do "Work Programme sobre Comércio Eletrônico", os Estados Unidos argumentaram que, enquanto a transmissão de produtos digitais, inclusive de um arquivo CAD, pode ser caracterizada como um serviço, os produtos em si não são consumidos ("used up") nessa transmissão, mas "mantêm permanência

\footnotetext{
15 Mais adiante mostra-se que os serviços são classificados, segundo a forma de prestação, por quatro modos distintos.

16 Canada-Certain Measures Concerning Periodicals, WT/DS31/AB/R, adotado em 30 de jun. 1997.

17 FLEUTER, Sam. The Role of Digital Products Under the WTO: A New Framework for GATT and GATS Classification. Chicago Journal of International Law, Vol. 17: n. 1, Article 5, 2016, p. 164. Disponível em: http://chicagounbound.uchicago.edu/cjil/vol17/ iss1/5. Acesso em: 5 de nov. 2018.
} 
análoga ao mundo dos bens". Assim, de acordo com essa visão, a maioria dos produtos digitais estaria mais bem enquadrada no GATT.

O problema com o uso de definições formalistas de bens e serviços é que ele se baseia, necessariamente, em um raciocínio indutivo. Essas definições podem ser úteis para delinear fronteiras sobre o que significa um bem ou serviço, mas não ajudam na classificação de um determinado produto. ${ }^{18}$

A partir de uma ótica que poderia ser denominada de funcional, uma tentativa de classificarem-se produtos digitais em bens ou serviços envolveria a análise de alguns dos princípios fundamentais da OMC. Um desses princípios é o da similaridade ("likeness"). De acordo com esse princípio, os Membros da OMC devem outorgar tratamento similar a bens ou serviços similares. Ao agregar-se a ele o Princípio da Neutralidade da Rede, reconhecido pelo Órgão de Apelação em China-Measures Affecting Certain Publications and Audiovisual Entertainment Product ${ }^{19}$, conclui-se que um arquivo digital não poderia ser tratado de forma diferente baseado no fato de que foi transportado por meio da Internet ou em um CD (se o CD tiver sido comprado diretamente de uma loja ou encomendado pela Internet). ${ }^{20}$

Considerando-se que "e-products", tais como "e-books" ou arquivos de música digital, possuem claramente similares físicos - livros impressos em papel e CDs - deveriam ser tratados como bens, sujeitos, portanto, ao GATTT. Porém, a aplicação do Princípio da Similaridade é menos óbvia quando os produtos digitais em questão são os arquivos em CAD. Em tese, seria possível considerar que um produto impresso em $3 \mathrm{D}$ deveria receber o mesmo tratamento que o produto acabado nos moldes convencionais. Assim, por exemplo, o projeto ("design") de uma peça de avião da Embraer (Empresa Brasileira de Aeronáutica S.A), contido no arquivo $\mathrm{CAD}$, teria o mesmo tratamento que as próprias peças. Ambos seriam bens sob o amparo do GATT.

\footnotetext{
18 FLEUTER, Sam. The Role of Digital Products Under the WTO: A New Framework for GATT and GATS Classification. Chicago Journal of International Law, Vol. 17: n. 1, Article 5, 2016, p. 165-166. Disponível em: http://chicagounbound.uchicago.edu/ cjil/vol17/iss1/5. Acesso em: 5 de nov. 2018.

19 China-Measures Affecting Certain Publications and Audiovisual Entertainment Products, WT/DS363/AB/R (relatório adotado em 21 dez. 2009). Consultar nota de rodapé n. ${ }^{\circ} 9$.

$20 \mathrm{Na}$ realidade, o Órgão de Apelação reconheceu o princípio da neutralidade tecnológica, mas falhou ao enunciar uma regra clara sobre como colocá-lo na prática.
}

Essa análise fundada no Princípio da Similaridade comporta, no entanto, o mesmo raciocínio indutivo limitador das definições formalísticas. Um sistema que se vale de comparações com o passado não parece adequado para tomar decisões baseadas em novos padrões factuais. O Princípio da Similaridade ignora que, inevitavelmente, a inovação tecnológica proporcionará o surgimento de produtos digitais que não terão similares físicos. ${ }^{21}$

O setor de serviços está no centro dessa inovação ou revolução tecnológica. Tecnologias digitais têm propiciado que uma crescente gama de serviços seja comprada "on line" e fornecida além-fronteiras. Ademais de facilitar o comércio em setores tradicionais, as tecnologias digitais implicam a substituição do comércio de bens por novos serviços A denominada "servicificação" da economia intensificou as áreas cinzentas entre o comércio de bens e serviços. Como salientado por Mira Burri, "[o]verall, the relationship between trade in goods and trade in services becomes more complex in the digital space; previous distinctions between goods and services may not be valid any longer and this has regulatory implications under current international trade law...". ${ }^{22}$

É razoável, assim, sustentar que a tecnologia 3D, que utiliza o CAD, constitua um serviço. Nesse caso, seria necessário verificar em qual dos quatro Modos de prestação de serviços, previstos no art. I, 2, do GATS, ela se enquadraria. Poderia ser no Modo 1, prestação transfronteiriça de serviços ("cross-border supply"), isto é, de um território de um Membro (da OMC) ao território de qualquer outro Membro, ou no Modo 2, consumo no exterior ("consumption abroad"), isto é, no território de um Membro aos consumidores de serviços de qualquer outro Membro. ${ }^{23}$

${ }^{21}$ FLEUTER, Sam. The Role of Digital Products Under the WTO: A New Framework for GATT and GATS Classification. Chicago Journal of International Law, Vol. 17: n. ${ }^{\circ}$ 1, Article 5, 2016, p. 168. Disponível em: http://chicagounbound.uchicago.edu/cjil/vol17/ iss1/5. Acesso em: 5 de nov. 2018.

22 BURRI, Mira. Understanding and Shaping Trade Rules for the Digital Era, p. 12. Disponível em: https://www.researchgate. net/publication/329419386_Understanding_and_Shaping_Trade_ Rules_for_the_Digital_Era. Acesso em 26 de mar. 2019.

23 Os outros dois Modos são: Modo 3, presença comercial, isto é, prestador de serviços de um Membro, por intermédio da presença comercial, no território de qualquer outro Membro, e Modo 4, presença de pessoas físicas, isto é,. prestador de serviços de um Membro, por intermédio da presença de pessoas físicas de um Membro no território de qualquer outro Membro. CELLI JUNIOR, Umberto. Comércio de Serviços na OMC: liberalização, condições e desafios. Curitiba, Juruá, 2009, p. 87. 
Apesar de suas normas serem produto do sistema GATT (i.e., foi feita uma adaptação para o GATS dos conceitos e princípios do GATT), o GATS tem maior abrangência . Essa abrangência deve ser examinada no contexto de uma estrutura que, além de complexa, é muito mais flexível. Essa flexibilidade permite aos Membros inscrever, em suas Listas de Compromissos, os setores e subsetores que desejam liberalizar ou estabelecer as restrições que entenderem necessárias. Ela é mais compatível com as incertezas quanto à classificação das tecnologias digitais. Permite aos Membros abrir os mercados de serviços, mas, ao mesmo tempo, mantê-los protegidos em alguma medida. Em princípio, portanto, a impressão em 3D com arquivo CAD estaria melhor ao abrigo do GATS.

De qualquer forma, para que compromissos pudessem ser assumidos, seria necessária a adoção pela OMC de um "cluster approach" para a classificação dos produtos digitais ou que os Membros tentassem chegar a um consenso sobre o escopo dos serviços abrangidos nos diferentes setores da Lista de Classificação Setorial em Serviços, a W/120. ${ }^{24}$ Em vez de desenvolver novas disciplinas, a atual estrutura do GATS poderia simplesmente ser usada para estimular os Membros a aperfeiçoar e a expandir seus compromissos nos vários setores relacionados a tecnologia.

Não seria tarefa fácil, contudo, acomodar os serviços digitais em uma classificação (a W/120) concebida há quase trinta anos. As plataformas digitais são multifuncionais e muito mais abrangentes e não se enquadrariam em um setor ou subsetor específico de serviços. ${ }^{25}$

\section{Blockchain}

O blockchain ganhou relevância inicialmente como uma plataforma que suporta a criptomoeda, o bitcoin. Atualmente, não se limita, apenas, ao bitcoin. ${ }^{26}$ Poderá

\footnotetext{
24 Referência mais específica à W/120 e aos setores e subsetores de serviços no item 4.1, abaixo.

25 Interessante discussão a respeito em: MITCHELL, Andrew D.; MISHRA, Neha. Data at Docks: Modernising International Trade Law for the Digital Economy. Vanderbilt Journal of Entertainment \& Technology Law, vol. 20, 2018, em especial p. 1090-1091 e 1126. Disponível em: https://ssrn.com/abstract=3064396, acesso em 5 de nov. 2018.

26 Poderá ser também ser útil em vários outros setores, como, por exemplo, o de mercados de carbono. Em interessante estudo a respeito dos mercados de carbono, PIGEOLET e WAEYENBERGE
}

vir a revolucionar o comércio internacional, tornando-o mais barato, rápido e simples, via cadeias globais de suprimento. Para ser revolucionária, no entanto, essa tecnologia precisa ser implantada globalmente, o que requer um quadro regulador internacional.

O blockchain constitui uma base de dados compartilhada em uma rede de computadores. É um tipo de "distributed ledger technology" (tecnologia de registro (livro de contas) distribuído), uma base eletrônica de dados descentralizada ${ }^{27}$ que, continuamente, registra, verifica e armazena blocos de informação em formato digital, assegurados por criptografia. As informações registradas referem-se a transações em ativos e troca de informações. Essencialmente, o blockchain envolve: (i) o registro (qualquer informação, como a de uma venda e pode incluir assinaturas digitais); (ii) o bloco (um pacote de registros); e (iii) uma cadeia (todos os blocos juntos).

Quando um novo registro é efetuado, a validade de seus detalhes é verificada pelos computadores na rede, denominados "nodes". Uma vez aceito, esse registro é adicionado a um bloco. Isso cria um registro permanente e cronológico de cada transação. Blocos que consistem em informação a respeito de uma ou mais transações são acrescidos à cadeia de blocos existentes. A criptografia mantém o blockchain seguro e resistente a

pontuam que "[the] blockchain is indeed an interesting tool whose mobilization seems necessary for the implementation of the political and legal framework of the fight against global warming." Especificamente, dizem eles, "the blockchain could be used to improve carbon trading; some argue for the free use of digital ledgers as a platform for networking carbon markets so as to achieve the objectives of the Paris Agreement". PIGEOLET, Louise; WAEYNBERGE, Araud Van. Assessment and Challenges of Carbon Markets. Revista de Direito Internacional (Brazilian Journal of International Law), vol. 16, n. ${ }^{\circ}$ 2, 2019, p. 86 .

27 Como descrito por De Filippi e Wright, “....blockchains are decentralized databases, maintained by a distributed network of computers. They blend together a variety of different technologies including peer-to-peer networks, public-private key cryptography and consensus mechanisms - to create a novel type of database". "Blockchain technology constitutes an infrastructure for the storage of data and the management of software applications, decreasing the need for centralized middlemen...Databases serve as a backbone for every platform, website, app, or other online service. Up to this point, databases have for the most part been maintained by centralized intermediaries, such as large Internet companies or cloud computing operators such as Amazon, Microsoft, and Google. Blockchains are changing this dynamic, powering a new generation of disintermediated peer-to-peer applications, which are less dependent on centralized control." DE FILIPPI, Primavera; WRIGHT, Aaron. Blockchain and the Law - The Rule of Code. Londres: Harvard University Press, 2018, p. 13 e 33. 
"hackers" ou a alteração não autorizada, o que lhe confere o caráter de imutabilidade, ou seja, transações podem ser adicionadas à cadeia, mas aquelas já registradas não podem ser apagadas. ${ }^{28}$

Como mencionado acima, um uso potencial do blockchain, associado ao comércio internacional, está relacionado às cadeias globais de suprimento. Uma cadeia de suprimento é essencialmente um conjunto de processos e recursos necessários à entrega final do produto ao consumidor. Ela envolve o fluxo de material (produtos físicos, material e suprimentos), de dinheiro (pagamentos, informações sobre cartões de crédito, pagamentos eletrônicos ("e-payments")) e de dados (informação relacionada à demanda, embarcações, pedidos, prazos, dentre outras).

Esse fluxo permite distinguir a natureza intrinsecamente sem fronteiras do blockchain. Qualquer legislação de um país que tenha o efeito de restringir em especial o fluxo de dados transfronteiriços pode constituir uma barreira ao desenvolvimento dessa tecnologia e seu uso em cadeias de suprimento. Leis de proteção de dados que requerem a localização de dados, isto é, que os dados sejam processados e armazenados localmente, proibindo a coleta ou a transferência de dados a outros países sem autorização governamental, são exemplos desse tipo de barreira ${ }^{29}$.

Essas leis podem ser abrangentes cobrindo a maioria ou todos os tipos de dados ou se concentrar em tipos específicos de dados, tais como exigências de que pagamentos sejam processados no próprio país. A localização de dados é incompatível com a típica logística multijurisdicional do blockchain que requer armazenamento de dados em uma rede descentralizada e não em uma jurisdição exclusiva.

O General Data Protection Regulation (“GDPR”) da União Europeia, em vigor desde 25 de maio de 2018, bem como a Lei Geral de Proteção de Dados brasileira, Lei No. 13.709, de 14 de agosto de $2018{ }^{30}$ ("LGPD”),

\footnotetext{
28 Mais detalhes sobre as características do blockchain em EPPS, Tracey; CAREY, Blake; UPPERTON, Tess. Revolutionizing Global Supply Chains One Block at a Time: Growing International Trade with Blockchain - Are International Rules Up to the Task? Society of International Economic Law (SIEL), Sixth Biennial Global Conference, 2018, p. 1-2. Disponível em: https://papers.ssrn.com/sol3/papers. cfm?abstract_id=3210386. Acesso em 8 de nov. 2018.

$29 \mathrm{Na}$ China, por exemplo, o governo restringiu tanto o fluxo interno como o transfronteiriço de informação e dados.

30 Alterada pela Lei n. ${ }^{\circ} .13 .853$, de 8 de jul. 2019, que, dentre out-
}

permitem a transferência internacional de dados para outros países, desde que estes apresentem nível considerado adequado de proteção. Se, de um lado, essa condição é importante no tocante à proteção à privacidade, de outro, ela pode constituir-se em obstáculo ao comércio internacional.

A depender do entendimento da Comissão, no caso da União Europeia, e da Autoridade Nacional de Proteção de Dados, no caso do Brasil, a transferência poderá não ocorrer, interrompendo e impedindo o fluxo de dados essencial ao blockchain e às cadeias globais de suprimento do comércio internacional. ${ }^{31}$

\subsection{Blockchain e o GATS}

Com base em uma interpretação extensiva, pode-se argumentar que, de alguma forma, as normas do GATS regulam e permitem os fluxos de dados transfronteiriços e o comércio de serviços digitais. Por essa razão, o GATS é de fundamental importância para o blockchain. ${ }^{32}$

ras modificações, cria a Autoridade Nacional de Proteção de Dados. A Lei n. ${ }^{\circ} 13.709$, de 14 de agosto de 2028, até a finalização deste artigo, ainda não havia entrado em vigor.

31 Apesar de o GDPR e a LGPD aplicarem-se, apenas, a dados pessoais, é importante ressaltar que, em ambos, a definição de dados pessoais abrange qualquer informação relacionada a uma pessoa identificada ou identificável. Isso poderia incluir dados como endereço de IP, de localização e outros fatores específicos. Por essa razão, assinalam Epps, Carey e Upperton, "[d]ata in a supply chain blockchain could risk falling under this definition and being subject to GDPR data requirements. Establishing a definition of "nonpersonal" data is difficult when personal data itself is amorphously defined, and currently it is not clear when the proposed Regulation would apply". EPPS, Tracey; CAREY, Blake; UPPERTON, Tess. Revolutionizing Global Supply Chains One Block at a Time: Growing International Trade with Blockchain - Are International Rules Up to the Task? Society of International Economic Law (SIEL), Sixth Biennial Global Conference, 2018, p. 10. Disponível em: https://papers. ssrn.com $/$ sol3/papers.cfm?abstract_id $=3210386$. Acesso em 8 de nov. 2018.

32 Além disso, o GATS "has a more promising case in providing a better foundation for covering blockchain technologies. Digital transactions do not generally include tangible products, and the sale of electronic products often requires the continual delivery of services - whether for support or for maintenance — for them to be even usable. Many electronic products are covered by GATS in that their advertising, payment, delivery and distribution all significantly involve an electronic service. Moreover, blockchain essentially involves the validation by users of transactions conducted over the platform. Whilst users cannot do so without their computing devices, blockchain still intuitively involves the supply of a service and not the sale of a useful electronic product." RAZON, Arvin Kristopher. Liberalizing Blockchain: An Application of the GATS Digital Trade Framework. Melbourne Journal of International Law, 6, 
De acordo com o GATS, os Membros da OMC podem assumir compromissos tendo por referência a "Lista de Classificação Setorial em Serviços (W/120)" (Services Sectoral Classification List) concebida no início dos anos 1990 e que contém uma relação de aproximadamente cento e sessenta setores e subsetores de serviços . Os Membros utilizam, ainda, a denominada "Lista de Classificação de Produto Central" (Central Product Classification - CPC), elaborada pela divisão de estatística da ONU igualmente no início da década de $90 .{ }^{33}$

O GATS antecede o conceito de fluxo de dados transfronteiriços. Na época de sua negociação, a Internet estava no seu limiar, apenas começando a ser utilizada comercialmente. Somente no início dos anos 2000, a Internet passou a permitir, efetivamente, que as empresas realizassem vendas e fornecessem serviços "on line". O rápido desenvolvimento da Internet e de outras tecnologias coloca, assim, a seguinte questão: como poderiam os Membros da OMC interpretar as definições ultrapassadas das Listas de Classificação acima mencionadas e os compromissos de liberalização assumidos? ?34 $^{34}$

Os subsetores de serviços de relevância para o blockchain são aqueles relacionados à rubrica "computer and related services" 35 , que incluem os subsetores "data processing services" e "data base services". Os compromissos em serviços de telecomunicações também são importantes. Observa-se, no entanto, sobreposição

2019. Disponível em: http://classic.austlii.edu.au/au/journals/MelbJIL/2019/6.html. Acesso em: 8 de mai. 2020.

33 "Os negociadores do GATS, em vez de se preocuparem com uma terminologia precisa, optaram pela seguinte classificação de serviços: (i) serviços de negócios ou empresariais; (ii) serviços de comunicação; (iii) serviços de construção; (iv) serviços de distribuição; (v) serviços educacionais; (vi) serviços ambientais; (vii) serviços financeiros; (viii) serviços relacionados à saúde e serviços sociais; (ix) serviços de turismo e relacionados a viagens; (x) serviços de recreação, serviços culturais e esportivos; (xi) serviços de transporte; e (xii) outros serviços." Trata-se, portanto, de onze setores subdivididos na W/120. "Com o propósito de obter maior clareza com relação aos compromissos assumidos, muitos Membros acresceram às suas listas os respectivos números $\mathrm{CPC}$ aos nomes dos setores. Ambas as classificações são de uso facultativo e servem apenas para orientação, podendo os Membros inscrever compromissos em setores e subsetores não mencionados nesses documentos." CELLI JUNIOR, Umberto. Comércio de Serviços na OMC: liberalização, condições e desafios. Curitiba, Juruá, 2009, p.86-87.

34 A última atualização da CPC foi feita em 2015 e refere-se à internet e serviços digitais, mas poderia prover melhores diretrizes para os Membros.

35 A classificação 'computer and related services' não era tão relevante como é atualmente e não reflete, portanto, a complexa realidade dos serviços digitais. entre essa categoria e a dos "computer services". Isso porque atividades como serviços de "database" e "data processing" são providos "on line" e, no caso do blockchain, têm de ser providos "on line".

É possível sustentar, portanto, que o blockchain estaria abrangido pelos compromissos de liberalização assumidos por alguns Membros, uma vez que é, essencialmente, uma forma de serviços de base de dados.

Além disso, o Anexo sobre Telecomunicações do GATS $^{36}$ estabelece que, quando os Membros tiverem assumido compromissos, eles devem assegurar que os provedores de serviços estrangeiros possam usar redes de transportes de telecomunicações para o movimento transfronteiriço de informações e para o acesso de informações contidas em bases de dados. Nesse sentido, como salientado no World Trade Report 2018, da OMC,

it is of particular significance to online activity and the incumbent data flows involved that the Annex addresses information transfers. It requires members to ensure that foreign service suppliers may use basic telecommunications for the movement of digitalized information both within and across borders, including for intra-corporate communications and for access to information contained in databases or otherwise stored in the territory of any member. All suppliers of committed services benefit from these obligations. ${ }^{37}$

Se o blockchain está abrangido pelos compromissos assumidos por alguns Membros nos termos do Anexo sobre Telecomunicações, a outra questão que surge é se as regras de localização de dados e de privacidade que

36 O Anexo sobre Telecomunicações aplica-se complementarmente ao GATS a todas as medidas que afetem o acesso às redes e serviços públicos de telecomunicações e sua utilização. De acordo com seu art. $5^{\circ}$, os Membros obrigam-se a prover aos prestadores de serviços de qualquer outro Membro acesso às suas redes públicas de transportes de telecomunicações e serviços, bem como sua utilização em termos e condições razoáveis e não discriminatórias. Os serviços de telecomunicações viabilizam e dão suporte aos fluxos de dados transfronteiriços. Os fluxos de dados transfronteiriços impulsionados por serviços básicos e de valor adicionado de telecomunicações, tais como processamento e armazenamento de dados por meio de alta capacidade (isto é, armazenamento em nuvem ("cloud storage"), permitem às empresas não apenas a venda de seus produtos e serviços, como também a coordenação de suas atividades logísticas. Serviços de acesso à Internet por banda larga e outras redes de dados oferecem as mais altas velocidades necessárias à exploração de tecnologias como computação nas nuvens que possibilitam o uso de serviços que requerem transferência de grandes quantidades de dados.

37 WTO. WTO World Trade Report (2018) - The future of World Trade: how digital technologies are transforming global commerce, p.153. Disponível em: https://www.wto.org/english/res_e/publications_e/world_ trade_report18_e.pdf. Acesso em: 20 de out. 2018. 
restringem o fluxo de dados transfronteiriços são inconsistentes com tais compromissos. Em princípio, seriam. Essas regras impedem o comércio de serviços de bases de dados, uma vez que estes não podem ocorrer se os dados não forem transferidos internacionalmente.

Haveria, em tese, também, um descumprimento da obrigação de tratamento nacional (proibição de tratar fornecedores estrangeiros de forma menos favorável que os nacionais) e possivelmente de acesso a mercado que pró́be restrições quantitativas a prestadores de serviços estrangeiros. De certa forma, o GDPR também poderia ser considerado inconsistente com os compromissos de acesso a mercados sob o GATS porque, como visto anteriormente, contém restrições ao fluxo transfronteiriço de dados. (A União Europeia, por exemplo, assumiu compromissos com relação aos serviços de bases de dados).

No caso dos poucos Membros que assumiram compromissos relevantes, é possível afirmar que tais compromissos asseguram a livre circulação transfronteiriça de dados, o que colocaria a OMC em boa posição para regular o blockchain. Deveria, porém, haver um reconhecimento explícito das intersecções do GATS com o blockchain, o que, como se sabe, ainda não ocorreu.

Como, até o momento, não houve uma formulação abrangente para uma resposta aos desafios trazidos pela era digital, o GATS foi, em certa medida, superado por outros acordos comerciais plurilaterais e bilaterais. Em especial, acordos de livre comércio como o "Comprehensive Progressive Transpacific Partnership Agreement" ("CPTPP”') parecem estar mais bem aparelhados para regular o blockchain, apesar da limitação de seu escopo territorial. ${ }^{38}$

Apesar de não dispor, expressamente, a respeito do uso da "distributed ledger technology", há no CPTPP um capítulo específico (capítulo 14) sobre comércio ele-

38 EPPS, Tracey; CAREY, Blake; UPPERTON, Tess. Revolutionizing Global Supply Chains One Block at a Time: Growing International Trade with Blockchain - Are International Rules Up to the Task? Society of International Economic Law (SIEL), Sixth Biennial Global Conference, 2018, p. 13-16. Disponível em: https://papers.ssrn.com/ sol3/papers.cfm?abstract_id=3210386. Acesso em 8 de nov. 2018. O CPTPP é composto por Austrália, Brunei, Canadá, Chile, Japão, Malásia, México, Nova Zelândia, Peru, Singapura e Vietnã e foi assinado em 8 de março de 2018, após a retirada dos Estados Unidos do anterior "Trans-Pacific Partnership Agreement" ("TPP”). O CPTPP incorpora o texto original do TPP, com exceção de aproximadamente 20 disposições que ficaram em suspenso até eventual posterior decisão contrária das partes. Entrou em vigor em 30 de dezembro de 2018. trônico que merece destaque. Estabelece o art. 14.11:

Art. 14.11: Cross-Border Transfer of Information by Electronic Means

1. The Parties recognise that each Party may have its own regulatory requirements concerning the transfer of information by electronic means.

2. Each Party shall allow the cross-border transfer of information by electronic means, including personal information, when this activity is for the conduct of the business of a covered person.

3. Nothing in this Article shall prevent a Party from adopting or maintaining measures inconsistent with paragraph 2 to achieve a legitimate public policy objective, provided that the measure:

(a) is not applied in a manner which would constitute a means of arbitrary or unjustifiable discrimination or a disguised restriction on trade; and

(b) does not impose restrictions on transfers of information greater than are required to achieve the objective. $^{39}$

Trata-se, essencialmente, o parágrafo segundo de uma proibição à limitação de exportação de dados relativamente à toda informação — e não apenas à informação pessoal. Essa proibição, contudo, está sujeita à ressalva do parágrafo terceiro, o qual autoriza as Partes a limitar ou restringir a exportação de dados desde que o façam, observadas certas condições, com o propósito de implantar legítima política pública. Não há especificação do que seja legítima política pública.

Essas disposições do CPTPP constituem um passo na direção certa no tocante à facilitação do desenvolvimento do blockchain associado ao comércio internacional. Mostram o efetivo potencial dos acordos de livre comércio em conciliar interesses divergentes como a proteção de dados, de um lado, e seu livre fluxo, de outro. ${ }^{40}$ Podem, por fim, servir de inspiração para negociações no âmbito da OMC, em que, há mais de 20 $\operatorname{anos}^{41}$, tenta-se, sem sucesso, elaborar regras sobre o comércio eletrônico.

39 Texto do CPTPP disponível em: https://www.mfat.govt.nz/ en/trade/free-trade-agreements/free-trade-agreements-in-force/ cptpp/comprehensive-and-progressive-agreement-for-trans-pacific-partnership-text/ Acesso em 6 de nov. 2018.

40 EPPS, Tracey; CAREY, Blake; UPPERTON, Tess. Revolutionizing Global Supply Chains One Block at a Time: Growing International Trade with Blockchain - Are International Rules Up to the Task? Society of International Economic Law (SIEL), Sixth Biennial Global Conference, 2018, p.16. Disponível em: https://papers.ssrn.com/ sol3/papers.cfm?abstract_id=3210386. Acesso em 8 de nov. 2018.

41 Desde a constituição do 'Work Programme on Electronic Commerce' em 1998. 
Nesse sentido, durante a Conferência Ministerial, realizada em Buenos Aires em dezembro de 2017, um grupo de 71 Membros comprometeu-se a elaborar trabalhos preliminares visando ao início efetivo de negociações a respeito. Em janeiro de 2019, no Fórum Econômico Mundial de Davos, novo Comunicado Conjunto foi concluído por 76 Membros, os quais confirmaram sua intenção de iniciar negociações comerciais com o propósito de alcançar resultados de alto padrão com a participação do maior número possível de Membros da OMC.42 Em maio de 2019, cerca de 19 Membros enviaram propostas para negociação de um acordo plurilateral sobre comércio eletrônico. Dentre os tópicos dessas propostas, aparecem proteção e localização de dados e transferência internacional de dados.

Trata-se de sinais positivos de engajamento na estruturação do comércio internacional regulado pelas novas tecnologias.

\section{Considerações finais}

Como visto, não há, ainda, uma classificação amplamente aceita dos modos de comércio que dependem dos meios digitais. Isso torna complicada qualquer tarefa de avaliação abrangente e bem-sistematizada das efetivas implicações das tecnologias digitais para o sistema multilateral de comércio e o papel da OMC como um todo. Daí a escolha inspirada no World Trade Report 2018 da OMC de duas tecnologias de crescente importância para o comércio internacional, a impressão em 3D e o blockchain, tendo como base alguns aspectos do GATT e do GATS, pois elas permitem algumas reflexões interessantes, embora não conclusivas.

No caso da impressão em 3D, a fundamental questão envolvendo a distinção entre bens e serviços não foi esclarecida. Em face da incontornável dificuldade em separá-los atualmente, tal como se fez até um passado recente, sua dicotomia regulatória GATT ou GATS, talvez, tenha perdido o sentido. ${ }^{43}$

\footnotetext{
42 O documento circulou como WT/L/1056.

43 Afinal, "goods are increasingly bundled with services; and new, and previously non-tradable, services are now being traded across borders”. GONZÁLEZ, Javier López; JOUANJEAN, Marie-Agnes. Digital Trade: Developing a Framework for Analysis, 2017, p. 8. Disponível em: https://www.oecd-ilibrary.org/trade/digitaltrade_524c8c83-en. Acesso em: 6 mai. 2020.
}

De qualquer forma, se ainda fizer algum sentido, se um produto originado da impressão em $3 \mathrm{D}$, como o contido no arquivo CAD transmitido para outro país por meio eletrônico, for considerado um bem - com todas as implicações tributárias decorrentes-, seria mais indicado que — além do GATT, claro —, estivesse abrigado no ITA. Para tanto, o ITA teria de contar com maior adesão dos Membros da OMC. Além disso, seria preciso atualizá-lo, modificando-se seu escopo e, consequentemente, sua lista de classificação que contempla, apenas, produtos físicos e não digitais.

Se, por outro lado, esse produto for considerado um serviço, seria necessária, no mínimo, uma reformulação da Lista de Classificação Setorial em Serviços (W/120) do GATS, considerando-se que as plataformas digitais são multifuncionais e muito mais abrangentes e não se enquadrariam em um setor ou subsetor específico de serviços.

Um caminho paralelo seria reativar as negociações plurilaterais do TISA. Lançado em 2013 com o propósito de aprofundar o acesso a mercados no setor de serviços, configura um espaço propício para fazer avançar as negociações sobre o alcance, a classificação e a regulação dos serviços digitais. ${ }^{44}$ O TISA é bastante "ambicioso e pretende englobar todos os setores de serviços, tais como serviços financeiros e serviços de tecnologia da informação, incluindo telecomunicação e e-commerce", entre outros. ${ }^{45}$ Não existe incompatibilidade entre o TISA e o GATS. A questão central é saber como o TISA poderá relacionar-se com a OMC. Constituirá um "building block" rumo a uma liberalização multilateral do comércio de serviços? ${ }^{46}$ Seja como for, como se trata de um acordo plurilateral, os Membros tendem a sentir-se mais estimulados a fazer maiores concessões nessa área.

Ao contrário da impressão digital em 3D, no caso

44 São partes negociadoras do TISA: Austrália, Canadá, Chile, Taipé Chinesa (Taiwan), Colômbia, Costa Rica, Hong Kong, Islândia, Israel, Japão, Liechtenstein, México, Nova Zelândia, Noruega, Paquistão, Panamá, Paraguai, Peru, Coreia do Sul, Suíça, Turquia, Estados Unidos e União Europeia. Está aberto à adesão de todos os Membros da OMC.

45 ROCHA GABRIEL; Vivien Daniele; LINHARES MESQUITA, Alebe. O Comércio de Serviços entre Brasil e Uruguai: liberalização, desafios e perspectivas do setor de tecnologia da informação e comunicação (TIC) e softwares. Revista de Direito Internacional (Bražilian Journal of International Law), vol. 13, n. ${ }^{\circ}$ 1, 2016, p. 67.

46 MARCHETTI, Juan A.; ROY, Martin. The TISA Initiative: An Overview of Market Access Issues. Journal of World Trade, vol. 48, n. ${ }^{\circ}$, 2014, p. 717. 
do blockchain, praticamente não existe essa dicotomia regulatória entre bem e serviço ${ }^{47}$. Ainda que as listas de classificação de serviços estejam ultrapassadas e necessitem de atualização, é plausível sustentar que o blockchain constitui um serviço de base de dados e, nessa condição, estaria abrangido pelos compromissos de liberalização assumidos por alguns Membros, especialmente nos termos do Anexo sobre Telecomunicações do GATS.

Esses compromissos e outros que eventualmente venham a ser assumidos asseguram a livre circulação transfronteiriça de dados, que é fundamental para alimentar as cadeias globais de suprimentos que caracterizam o blockchain. Isso coloca a OMC, pelo menos em tese, em boa posição para regular essa tecnologia digital de maneira mais específica. No entanto, para maior segurança jurídica, deveria haver, de alguma forma, reconhecimento explícito das intersecções do GATS com o blockchain.

Seja no GATS ou em um eventual acordo plurilateral sobre comércio eletrônico, não pode haver restrições à transferência internacional de dados. Qualquer restrição nesse sentido somente poderia ser adotada pelos Membros para implantar alguma política pública relevante, desde que esta não constitua um meio de arbitrária ou injustificável discriminação ou limitação disfarçada ao comércio internacional.

As questões regulatórias colocadas pelas tecnologias digitais impressão em 3D e o blockchain, tendo como pano de fundo o GATT e o GATS, apenas dão uma ideia dos enormes desafios que se impõem à necessária reformulação das regras do comércio global. A natureza ainda difusa e não totalmente compreendida do universo digital, bem como o constante processo de evolução das tecnologias que o envolvem, tornam bastante complexo um possível novo desenho normativo desse comércio global.

\section{Referências}

ASHTON-HART, Nick. Addressing the Networked Economy in Trade Policy. In: BRAGA, Carlos A.; HOEKMAN, Bernard (ed.). Future of the Global Trade

\footnotetext{
47 A natureza intrinsecamente digital do blockchain é um importante fator para sua caracterização mais como um serviço do que um bem.
}

Order. Florença, Lausanne, São Paulo: European University Institute, IMD, Fundação Dom Cabral, $2^{a}$ ed., 2017.

BURRI, Mira. Understanding and Shaping Trade Rules for the Digital Era. Disponível em: https://www. researchgate.net/publication/329419386_Understanding_and_Shaping_Trade_Rules_for_the_Digital_Era. Acesso em: 26 de mar. 2019.

CELLI JUNIOR, Umberto. Comércio de Serviços na OMC: liberalização, condiçoes e desafios. Curitiba: Juruá, 2009.

CIURIAK, Dan; PTASHKINA, Maria. Começaram as guerras no comércio digital: delineando os campos da batalha regulatória. Revista Pontes, vol. 14, n. ${ }^{\circ}$ 1, 2018. Disponível em: http://www.ictsd.org/bridges-news/ pontes $/$ news $/$ come $\% \mathrm{C} 3 \% \mathrm{~A} 7$ aram-as-guerras-nocom $\%$ C $3 \%$ A 9 rcio-digital-delineando-os-campos-debatalha. Acesso em: 29 de mar. 2019.

DE FILIPPI, Primavera; WRIGHT, Aaron. Blockchain and the Law - The Rule of Code. Londres: Harvard University Press, 2018.

EPPS, Tracey; CAREY, Blake; UPPERTON, Tess. Revolutionizing Global Supply Chains One Block at a Time: Growing International Trade with Blockchain Are International Rules Up to the Task? Society of International Economic Law (SIEL), Sixth Biennial Global Conference, 2018. Disponível em: https://papers.ssrn.com/ sol3/papers.cfm?abstract_id=3210386. Acesso em 8 de nov. 2018.

FLEUTER, Sam. The Role of Digital Products Under the WTO: A New Framework for GATT and GATS Classification. Chicago Journal of International Law, Vol. 17: n. ${ }^{\circ}$ 1, Article 5, 2016. Disponível em: http://chicagounbound.uchicago.edu/cjil/vol17/iss1/5. Acesso em: 5 de nov. 2018.

GONZÁLEZ, Javier López; JOUANJEAN, MarieAgnes. Digital Trade: Developing a Framework for Analysis, 2017. Disponível em: <https://www.oecdilibrary.org/trade/digital-trade_524c8c83-en>. Acesso em: 6 de mai. 2020.

MARCHETTI, Juan A.; ROY, Martin. The TISA Initiative: An Overview of Market Access Issues. Journal of World Trade, vol. 48, n. ${ }^{\circ}$ 4, 2014.

MITCHELL, Andrew D.; MISHRA, Neha. Data at Docks: Modernising International Trade Law for the Digital Economy. Vanderbilt Journal of Entertainment \& 
Technology Law, vol. 20, 2018. Disponível em: https:// ssrn.com/abstract $=3064396$. Acesso em: 5 nov. 2018.

OECD. The impact of digitalization on trade. Disponível em: https://www.oecd.org/trade/topics/digital-trade/. Acesso em: 8 de mai. 2020.

PARDOLESI, Roberto; DAVOLA, Antonio. What is wrong in the debate about Smart Contracts. Disponível em: https://papers.ssrn.com/sol3/papers. cfm?abstract_id=3339421. Acesso em: 5 de mai. 2020.

PIGEOLET, Louise; WAEYNBERGE, Araud Van. Assessment and Challenges of Carbon Markets. Revista de Direito Internacional (Brazilian Journal of International Law), vol. 16, n. ${ }^{\circ}$ 2, 2019.

RAZON, Arvin Kristopher. Liberalizing Blockchain: An Application of the GATS Digital Trade Framework. Melbourne Journal of International Law, 6, 2019. Disponível em: http://classic.austlii.edu.au/au/journals/ MelbJIL/2019/6.html. Acesso em: 8 de mai. 2020.

RIBEIRO ALVES, Gleisse. O Acordo GATS e sua Aplicação aos Serviços do Comércio Eletrônico. Revista de Direito Internacional (Brazilian Journal of International Law), vol. 12, n. ${ }^{\circ}$ 2, 2014.

ROCHA GABRIEL; Vivien Daniele; LINHARES MESQUITA, Alebe. O Comércio de Serviços entre Brasil e Uruguai: liberalização, desafios e perspectivas do setor de tecnologia da informação e comunicação (TIC) e softwares. Revista de Direito Internacional (Brazilian Journal of International Law), vol. 13, n. ${ }^{\circ}$ 1, 2016.

VOON, Tania. A New Approach to Audiovisual Products in the WTO: Rebalancing GATT and GATS. UCLA Entertainment Review, 14 (1), 2007.

WTO. WTO World Trade Report (2018) - The future of World Trade: how digital technologies are transforming global commerce. Disponível em: https://www.wto.org/english/res_e/publications_e/world_trade_report18_e. pdf. Acesso em: 20 de out. 2018.

WUNSCH-VINCENT, Sacha; HOLD, Arno. Towards coherent rules for digital trade: Building on efforts in multilateral versus preferential trade negotiations. In: BURRI, Mira; COTTIER, Thomas (Orgs.). Trade Governance in the Digital Age. Cambridge: Cambridge University Press, 2012. 
Para publicar na Revista de Direito Internacional, acesse o endereço eletrônico www.rdi.uniceub.br ou www.brazilianjournal.org.

Observe as normas de publicação, para facilitar e agilizar o trabalho de edição. 